\title{
Whole Packet Forwarding: Efficient Design of Fully Adaptive Routing Algorithms for Networks-on-Chip
}

\author{
Sheng Ma ${ }^{\dagger \ddagger}$, Natalie Enright Jerger ${ }^{\ddagger}$, Zhiying Wang ${ }^{\dagger}$ \\ ${ }^{\dagger}$ School of Computer, National University of Defense Technology, Changsha, China \\ ${ }_{\ddagger}^{\ddagger}$ Department of Electrical and Computer Engineering, University of Toronto, Toronto, Canada \\ masheng@nudt.edu.cn, enright@eecg.toronto.edu,zywang@nudt.edu.cn
}

\begin{abstract}
Routing algorithms for networks-on-chip (NoCs) typically only have a small number of virtual channels (VCs) at their disposal. Limited VCs pose several challenges to the design of fully adaptive routing algorithms. First, fully adaptive routing algorithms based on previous deadlockavoidance theories require a conservative $V C$ re-allocation scheme: a VC can only be re-allocated when it is empty, which limits performance. We propose a novel VC reallocation scheme, whole packet forwarding (WPF), which allows a non-empty VC to be re-allocated. WPF leverages the observation that the majority of packets in NoCs are short. We prove that WPF does not induce deadlock if the routing algorithm is deadlock-free using conservative $V C$ re-allocation. WPF is an important extension of previous deadlock-avoidance theories. Second, to efficiently utilize WPF in VC-limited networks, we design a novel fully adaptive routing algorithm which maintains packet adaptivity without significant hardware cost. Compared with conservative VC re-allocation, WPF achieves an average $88.9 \%$ saturation throughput improvement in synthetic traffic patterns and an average $21.3 \%$ and maximal $37.8 \%$ speedup for PARSEC applications with heavy network loads. Our design also offers higher performance than several partially adaptive and deterministic routing algorithms. ${ }^{1}$
\end{abstract}

\section{Introduction}

Networks-on-chip (NoCs) have been proposed to meet the communication requirements of many-core computing platforms [7]. NoC performance is sensitive to the choice of routing algorithm, as the routing algorithm defines not only the packet transmission latency, but also the saturation throughput a NoC can sustain. Many novel routing algorithms have been proposed to deliver high performance in NoCs [19, 21, 24, 28, 31, 43, 50].

\footnotetext{
${ }^{1}$ This research was carried out while Sheng Ma was a visiting international student at the University of Toronto supported by a CSC scholarship.
}

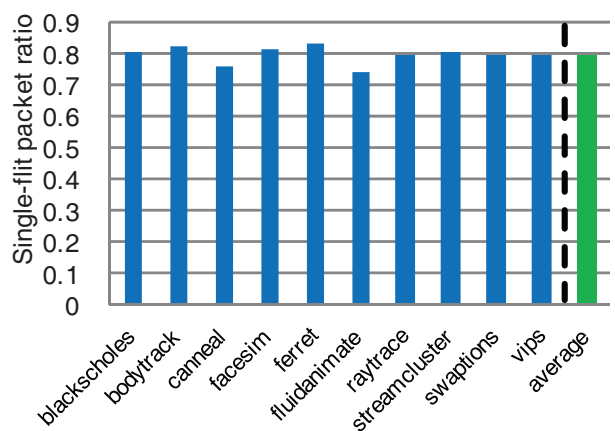

Figure 1. Single-flit packet ratio for the PARSEC benchmarks. (Flit width is 16 bytes.)

In addition to performance considerations, the routing algorithm has correctness implications for the network. Since deadlock is unacceptable, any proposed routing algorithm must be deadlock free, at both the network- and protocollevel. The guarantee of network-level deadlock freedom for a routing algorithm is generally based on deadlockavoidance theories. There are many theories for deadlockfree fully adaptive $[12,13,18,29,41,48]$ and partially adaptive routing algorithm design $[4,6,19,20]$. Although most theories were originally proposed for off-chip networks, they are widely used in today's NoCs $[19,21,24$, $28,31,43,50]$.

However, the characteristics of packets in NoCs are quite different than those in off-chip networks. Abundant wiring resources lead to wider flits which decreases the number of flits per packet; short packets dominate traffic in NoCs. In contrast, the wiring resources in off-chip networks are limited by the pin count. For example, the flit width of a typical off-chip router is on the order of 32 bits (e.g. the Alpha 21364 router [35]), while the flit width of a NoC is typically between 128 [22] and 256 bits [10]. With such wide flits, coherence messages carrying a memory address and control information but no data can be encoded as single-flit packets in NoCs. Figure 1 shows that on average $78.7 \%$ of packets are single flits for PARSEC benchmarks [3]; the remaining packets are 5 flits long and contain a full 64B cache line. 
Another noteworthy difference is that the buffer resources in NoCs are more precious than in off-chip networks due to the tight area and power budgets [17, 23], thus NoCs generally use flit-based wormhole flow control [9]. Although buffer resources are limited, several separate physical or virtual networks are leveraged for delivering different types of messages to avoid protocol-level deadlock. Table 1 lists the number of separate physical and virtual networks deployed in some industrial designs of offchip and on-chip networks. We also show the number of required virtual networks for some cache coherence protocols in the GEMS simulator [34]. Typically, four or five virtual networks are needed to avoid protocol-level deadlock. Considering the expense of buffers in NoCs, each virtual network will be configured with a small number of VCs [5] since more VCs require more buffers and a larger allocator. For example, TILE64 [49] and TRIPS [22] have only one $\mathrm{VC}$ per virtual network. Thus, a NoC routing algorithm is generally running with a limited number of VCs.

In a VC-limited network with short packets dominating traffic, the design of fully adaptive routing algorithms faces several new challenges. In a wormhole network, fully adaptive routing algorithms based on existing theories require a conservative $\mathrm{VC}$ re-allocation scheme: a $\mathrm{VC}$ can only be re-allocated to a new packet when it is empty $[12,13,18,29,41,48]$. This conservative scheme prevents network-level deadlock, but it is very restrictive resulting in bandwidth and performance loss in the presence of many back-to-back short packets [19]. Figure 2 illustrates the performance of three algorithms when each virtual network is configured with two $\mathrm{VCs}^{2}$. Despite its flexibility and load-balancing capability, the fully adaptive routing algorithm has even poorer performance than the deterministic and partially adaptive ones, since both the deterministic and partially adaptive algorithms can apply an aggressive $\mathrm{VC}$ re-allocation scheme. It is imperative to improve deadlock-avoidance theories to enhance the performance of fully adaptive routing algorithms in NoCs.

We propose a novel VC re-allocation scheme: whole packet forwarding (WPF), for fully adaptive routing algorithms. This scheme is summarized as follows: if a nonempty VC has enough buffer slots to hold the whole packet, then this VC can be re-allocated to the new packet even though it is not empty. WPF can be viewed as applying packet-based flow control in a wormhole network. This hybrid flow control mechanism solves the shortcoming of conservative VC re-allocation by allowing a VC to be reallocated before it is empty, which greatly improves the saturation throughput of fully adaptive routing algorithms. We prove that a fully adaptive routing algorithm using WPF is deadlock-free if this routing algorithm is deadlock-free with

\footnotetext{
${ }^{2}$ See Section 5 for detailed experimental configuration and description of the routing algorithms.
}

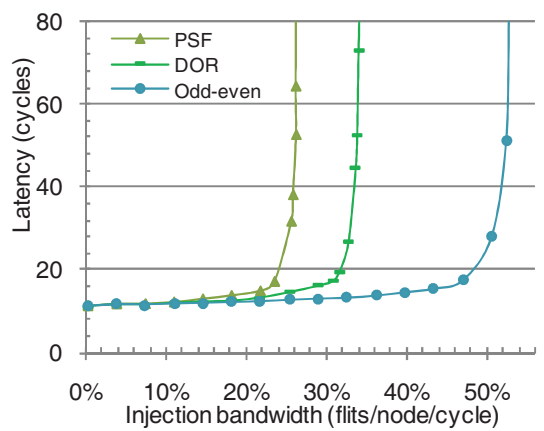

Figure 2. Routing algorithms performance with bit reverse traffic. (PSF: fully adaptive routing, DOR: deterministic routing; Odd-even: partially adaptive routing.)

conservative VC re-allocation. WPF is an important extension to previous deadlock-avoidance theories.

WPF enables the design of a fully adaptive routing algorithm with superior performance in a VC-limited network. Our novel routing algorithm achieves high VC utilization and maximal routing flexibility. Compared with conservative VC re-allocation, WPF provides an average saturation throughput improvement of $88.9 \%$ for synthetic traffic, and achieves an average $21.3 \%$ and maximal $37.8 \%$ speedup for PARSEC applications that heavily load the network. Our design also offers higher performance than several partially adaptive and deterministic routing algorithms. In summary, this paper makes the following primary contributions:

- Proposes WPF, which greatly improves the performance of fully adaptive routing algorithms, especially with limited VC resources.

- Proves WPF can be used by most previous deadlockfree fully adaptive routing algorithms; it is an important extension to existing deadlock-avoidance theories.

- Demonstrates that in a VC-limited network, maintaining packet adaptivity is very important and proposes an efficient fully adaptive routing algorithm that takes advantage of WPF.

\section{Background}

In this section, we discuss related work in deadlockavoidance theories and design methodologies for fully adaptive routing algorithms.

\subsection{Deadlock Avoidance Theories}

Since NoCs typically use wormhole flow control [9] to reduce buffering requirements [7, 33, 39], we focus on theories for wormhole networks. Dally and Seitz proposed a seminal deadlock avoidance theory [6] which can be used to design partially adaptive and deterministic routing algorithms. Duato introduced the concept of a routing subfunction, and gave an efficient design methodology [12, 13]. Lin et al. [29] leveraged the message flow model, and Schwiebert and Jayasimha [41] utilized the channel waiting 
Table 1. Number of physical/virtual networks. (PN: physical network; VN: virtual network)

\begin{tabular}{|l|l|l|l|l|l|}
\hline \multicolumn{3}{|c|}{ Industrial products } & \multicolumn{3}{c|}{ Cache coherence protocols in GEMS simulator [34] } \\
\hline Alpha 21364 [35] & TILE64 [49] & TRIPS [22] & MESI directory & MOESI directory & MOESI token \\
\hline 1 PN (7 VNs) & 5 PNs (1 VN/PN) & 2 PNs (4 VNs for OCN, 1 VN for OPN) & 5 VNs & 4 VNs & 4 VNs \\
\hline
\end{tabular}

graph to analyze deadlock properties. Recently, Verbeek and Schmaltz [47, 48] proposed a necessary and sufficient condition for deadlock-free routing based on static conditions. These theories $[12,13,18,29,41,48]$ can be used to design fully adaptive routing algorithms.

A limitation of these theories for fully adaptive routing is that they all require that a $\mathrm{VC}$ be re-allocated only when it is empty [12, 13, 18, 29, 41, 48]. This requirement guarantees that all blocked packets can reach the head of a VC to gain access to the 'deadlock-free' path at every router. However, considering the large fraction of short packets in NoCs, strict adherence to this requirement strongly limits performance, especially when the number of VCs is small. To address this issue, some deadlock-recovery based designs [1] or theories [15] are proposed, which remove the constraint of conservative VC re-allocation. They allow the formation of deadlocks, and then apply some recovery mechanism $[1,15]$. In contrast, WPF extends existing deadlock-avoidance theories which prohibit the formation of deadlock. To the best of our knowledge, WPF is the first proposal which allows multiple packets to reside in a $\mathrm{VC}$ concurrently for routing algorithms based on these previous deadlock-avoidance theories [12, 13, 18, 29, 41, 48].

Several partially adaptive algorithms based on the turn model have been proposed: negative-first, north-last, westfirst [20] and odd-even [4]. The Abacus turn model is a dynamically reconfigurable routing algorithm [19]. These partially adaptive algorithms allow aggressive VC reallocation: a VC can be re-allocated as soon as the tail flit of the last packet arrives [8]. This property can be directly deduced from Dally and Seitz's theory [6] since the channel dependency graphs of these algorithms are acyclic. However, they all suffer from limited adaptivity: packets cannot use all minimal paths between the source and destination, while fully adaptive ones can use all minimal paths.

\subsection{Fully Adaptive Routing Algorithms}

Duato's theory $[12,13]$ is widely used in the design of fully adaptive routing algorithms. In this theory, VCs are classified into two types: escape and adaptive. In the event of deadlock among the adaptive VCs, packets must have the opportunity to 'escape' to a deadlock-free set of VCs, known as escape VCs. Escape VCs are kept deadlock free by applying a more restrictive routing algorithm; dimension order routing (DOR) is typically used. An escape VC can only be used by a packet whose output port selection corresponds to a DOR path.

Many algorithms based on Duato's theory [21, 31, 35, 50] are composed of two parts: the routing function and selection strategy. If the selection strategy selects one output port, the packet can only request VCs that belong to the chosen output port. This requirement imposes a limitation on these algorithms: once a packet enters an escape $\mathrm{VC}$, it can only use escape VCs until it is delivered. Otherwise, the escape VC may be involved in deadlock. In a VC-limited network, such as a cache-coherent NoC, this limitation easily results in adaptivity loss. However, Duato's theory supports the design of algorithms which can use an adaptive VC after using an escape $\mathrm{VC}$ if packets are always guaranteed to be able to use escape VCs $[12,13]$. Based on these observations, we propose a design which maintains high adaptivity for packet routing, works well in a VC-limited environment and has low hardware overhead.

\section{Motivation}

In this section, we analyze the requirements of fully adaptive routing algorithms. We also illustrate how these requirements negatively affect performance.

\subsection{VC Re-allocation Scheme}

One limitation of fully adaptive routing algorithms is that at any time, a VC can hold at most one packet; a VC can only be re-allocated when it is empty. This is a reasonable requirement since fully adaptive routing algorithms put no limitation on the routing of some VCs and allow a cycle to form among VCs. For example, in routing algorithms based on Duato's theory, the adaptive VCs can be arbitrarily used $[12,13]$. If multiple packets are allowed to reside in the same $\mathrm{VC}$, a deadlock configuration is easily formed. Figure 3 illustrates a deadlock configuration [15]. Here each VN is configured with two VCs: an adaptive VC (AVC) and an escape VC (EVC). Configuring more VCs cannot eliminate this deadlock scenario since cycles are allowed to existed among adaptive VCs.

Eight packets are involved in this deadlock: $P_{0}-P_{7}$. The head flit of $P_{0}$ is behind the tail flit of $P_{1}$ in $A V C_{1}$. The same is true for $P_{1}, P_{2}, P_{4}, P_{5}$ and $P_{6}$. Although the head flits of $P_{3}$ and $P_{7}$ are at the head of $A V C_{3}$ and $A V C_{6}$, they cannot move forward as the two valid output VCs, $A V C_{0}$ and $E V C_{0}$, are both occupied by other packets. No packet can move forward. This deadlock is due to that some head flits are not at the VC heads, resulting some packets unable to gain access to the 'deadlock-free' path. Also, the tail flits of these packets resides in other VCs, prohibiting following packets to reach the head of these VCs or even utilize these VCs. The following packets then may cyclically block aforementioned packets. For example, the tail flit of $P_{0}$ resides in $A V C_{0}$, blocking $P_{3}$ from utilizing this $\mathrm{VC}$, which cyclically blocks the routing of $P_{0}$. 


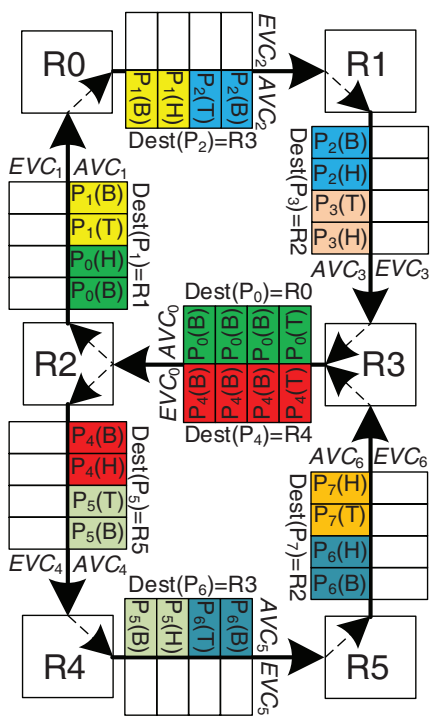

Figure 3. Deadlock in a fully adaptive routing algorithm if multiple packets are allowed to reside in one VC. (AVC: adaptive VC; EVC: escape VC; $P_{i}(\mathrm{H}), P_{i}(\mathrm{~B})$ and $P_{i}(\mathrm{~T})$ : the header, body and tail flit of Packet $P_{i}$, respectively; $\operatorname{Dest}\left(P_{i}\right)$ : the destination of Packet $P_{i}$.)

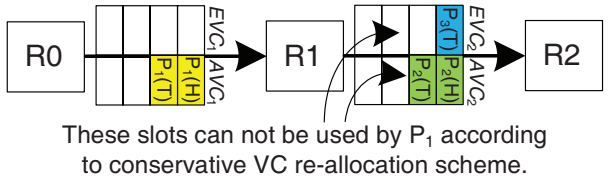

Figure 4. A VC underutilization scenario with conservative VC re-allocation. (AVC: adaptive VC; EVC: escape VC; $P_{i}(\mathbf{H})$ and $P_{i}(\mathbf{T})$ : the head and tail flit of Packet $P_{i}$.)

If the packet length is greater than the $\mathrm{VC}$ depth, allowing multiple packets to reside in one $\mathrm{VC}$ easily results in deadlock. When a long packet enters a non-empty VC, its header flit is not at the head of a VC, while its tail flit blocks the head of another VC. However, due to the abundant wiring on chip, NoC traffic is dominated by short packets. With many short or single-flit packets, strictly adhering to the requirement that a $\mathrm{VC}$ can only hold one packet reduces throughput and results in $\mathrm{VC}$ underutilization. In Figure 4, neither $E V C_{2}$ nor $A V C_{2}$ are available for reallocation; $P_{1}$ must wait in $A V C_{1}$ until either $E V C_{2}$ of $A V C_{2}$ becomes empty. However, since $P_{1}$ consists of only two flits, and both $E V C_{2}$ and $A V C_{2}$ have enough slots to hold this packet, forwarding $P_{1}$ into these VCs will not prevent following packets from getting to the head of $A V C_{1}$, as was the case in Figure 3. This is an opportunity for performance optimization. We will prove that $P_{1}$ can be forwarded into $E V C_{2}$ or $A V C_{2}$ without leading to deadlock.

\subsection{Packet Adaptivity}

In this section, we focus on maintaining packet adaptivity in VC-limited environments. Many fully adaptive routing algorithms based on Duato's theory are composed of

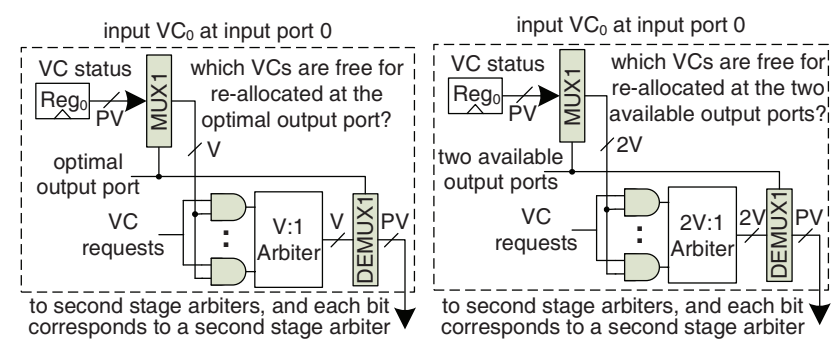

(a) Port-selection-first design.

(b) Naive design.

Figure 5. The structure of a first stage arbiter in a VC allocator for one VN. (Each VN is configured with $V$ VCs and $P$ input/output ports.)

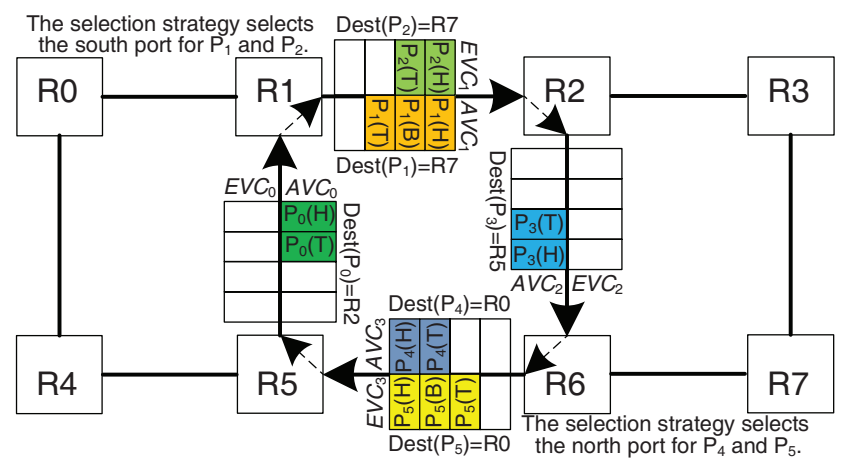

Figure 6. A deadlock configuration if packets in EVCs can apply for AVCs in port-selection-first algorithms.

two parts: routing function and selection strategy [21, 31, $35,50]$. The routing function computes all available output ports, then the selection strategy selects one of them. Once the selection strategy makes a choice, the packet can only request VCs for this particular port. This type of routing algorithm is called port-selection-first. Assuming a separable VC allocator consisting of two stages of arbiters [2, 36, 40], a port-selection-first algorithm only requires $V: 1$ arbiters in the first stage as shown in Figure 5(a).

A limitation of these algorithms is that once a packet enters an escape $\mathrm{VC}$, it must continue to use escape VCs; the packet will lose adaptivity in subsequent hops. Violating this limitation results in deadlock as shown in Figure 6. In this example, both south and east ports are available for $P_{1}$ and $P_{2}$. If the selection strategy chooses the south port, $P_{1}$ and $P_{2}$ can only apply for $A V C_{2}$. They cannot request $E V C_{2}$ because escape VCs can be only used when the chosen port adheres to DOR. Similarly, the selection strategy chooses the north port for $P_{4}$ and $P_{5}$; they can only apply for $A V C_{0}$. No packet can move forward. Thus, the limitation that once a packet enters an escape VC, it can only use escape VCs until delivered is necessary for port-selectionfirst algorithms. However, this requirement results in significant adaptivity loss with limited VCs, since packets have a high probability of going into escape VCs.

Duato's theory supports the design of algorithms which allows a packet to use adaptive VCs after using escape VCs, 
if it satisfies the following condition: a packet must be able to request an escape VC at any time. Once satisfy this condition, packets can always find a path whose VCs are not involved into cyclic dependencies since the extended channel dependency graph of escape VCs is acyclic $[12,13]$. To achieve this target, a packet could be allowed to request all available output VCs, since at least one output port must adhere to DOR and the packet can use the escape VC of this port $[12,13]$. However, this naive design results in additional hardware overhead. As shown in Figure 5(b), the VC allocator must use $2 \mathrm{~V}: 1$ arbiters in the first stage to cover the at most two available output ports for minimal routing algorithms. Based on these observations, we propose a novel design which maintains significant packet adaptivity with only minor additional hardware.

\section{Whole Packet Forwarding and Fully Adap- tive Routing}

In this section, we present our whole packet forwarding scheme and prove it is deadlock-free. Next, we design a routing algorithm which maintains packet adaptivity without significant hardware costs. Finally, we describe the hardware design and overhead.

\subsection{Whole Packet Forwarding}

As described in Section 3.1, existing fully adaptive routing algorithms use conservative $\mathrm{VC}$ re-allocation to prevent deadlock. However, this results in poor VC utilization. Therefore, we propose a novel VC re-allocation scheme: whole packet forwarding, which greatly improves VC utilization while not inducing deadlock. Suppose a packet $P_{k}$ with length of length $\left(P_{k}\right)$ currently resides in $V C_{i}$, and $V C_{j}$ is downstream of $V C_{i}$. Assume that the routing algorithm allows packet $P_{k}$ to use $V C_{j}$. With conservative VC re-allocation, $V C_{j}$ can be re-allocated to $P_{k}$ only if the tail flit of its most recently allocated packet has been sent out, i.e., it is currently empty [8]. For our proposed $\mathrm{VC}$ re-allocation scheme, $V C_{j}$ can be re-allocated if it already holds the tail flit of the most recently allocated packet, and the current free buffer count (free_slots $\left(V C_{j}\right)$ ) is greater than or equal to length $\left(P_{k}\right)$. If free_slots $\left(V C_{j}\right) \geq$ length $\left(P_{k}\right)$, then all flits of $P_{k}$ are guaranteed to be sent to $V C_{j}$ after a limited time ${ }^{3}$. We call this VC re-allocation scheme whole packet forwarding (WPF).

Figure 7 shows a WPF example. Here, the routing algorithm allows $P_{1}$ to use $V C_{2} . V C_{2}$ has already received the tail flit of $P_{2}$ and its free buffer count is two; this space is sufficient to hold the whole packet $P_{1}$ which consists of two flits. As a result, if we use WPF to re-allocate $V C_{2}$ to $P_{1}$, all flits of $P_{1}$ will be sent to $V C_{2}$ in a limited time. WPF forwards a packet into a non-empty VC

\footnotetext{
${ }^{3}$ The time to send all flits of $P_{k}$ to $V C_{j}$ will be determined by the congestion and switch allocation but all flits of $P_{k}$ are guaranteed to advance.
}

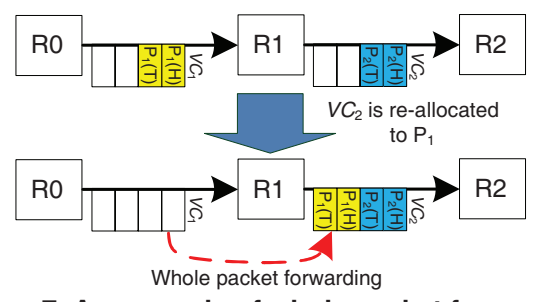

Figure 7. An example of whole packet forwarding.

if it has enough free buffers to hold the whole packet. In this case, WPF works similarly to packet-based flow controls such as store-and-forward (SAF) [16] and virtual cutthrough (VCT) [26]. However, if the downstream VC is empty, we still use wormhole flow control, which does not require the empty VC to have enough slots to hold the whole packet; this reduces the buffering requirements compared to SAF and VCT. WPF can be viewed as applying packetbased flow control in a wormhole network. This hybrid flow control mechanism solves the shortcoming of conservative VC re-allocation for fully adaptive routing algorithms.

Our contention is that if the routing algorithm with conservative VC re-allocation is deadlock-free, then applying WPF to forward packets into non-empty VCs will not lead to deadlock. If the VC depth is larger than the maximum packet length, and the network applies packet-based flow controls, multiple packets are allowed to reside in one VC for fully adaptive routing algorithms [14]. However, for the wormhole network, a blocking packet may reside in multiple VCs, introducing two additional dependencies, indirect and cross indirect dependency, between non-neighboring channels $[12,13,14]$. With these additional dependencies, it is difficult to prove the deadlock-free property of WPF based on existing theories.

We first give a qualitative proof for algorithms based on Duato's theory: using WPF will never allow a packet to get stuck 'mid-way' between two routers, as packets will always either be able to be fully transmitted to non-empty VCs or otherwise they will be able to use the escape VCs. However, the 'escape VC' is only defined in Duato's theory, and other theories may not have this definition. Thus, we provide a general proof. For convention, we label the routing algorithm with conservative $\mathrm{VC}$ re-allocation as $\mathrm{Alg}$; $A l g+W P F$ is $A l g$ with WPF applied to allow forwarding of entire packets to non-empty VCs.

Theorem 1: If $A l g$ is deadlock-free, then $A l g+W P F$ is also deadlock-free.

Informal Description: Our proof is by contradiction. We prove that if there is a deadlock configuration for $\mathrm{Alg}+$ $W P F$, then there is a deadlock configuration for $A l g$ as well. Using the deadlock configuration Config 0 shown in Figure 8 as an example, we remove these packets whose head flits are not at the heads of VCs, and get a new configuration Config. . We prove that $A l g$ can achieve Config $g_{1}$, and $\mathrm{Config}_{1}$ is a deadlock configuration. However, $\mathrm{Alg}$ is 


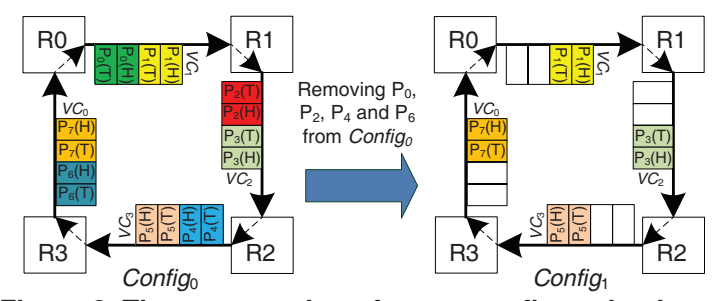

Figure 8. The construction of a new configuration based on Config of $A l g+W P F$.

deadlock-free, thus there is no such configuration.

Proof: By contradiction. If $\mathrm{Alg}+W P F$ is not deadlockfree, then there is a deadlock configuration (Config C $_{0}$ in which a set of packets, $P_{\text {set }}$ are waiting on VCs held by other packets in $P_{\text {set }_{0}}$. We prove that a deadlock configuration also exists for Alg. Our proof consists of three steps.

Step 1: We build a new configuration based on Config. Consider each packet $P_{i}$ in $P_{\text {set }}$. If $P_{i}$ is a packet whose header flit is not at the head of a VC, then this VC was allocated to $P_{i}$ using WPF; therefore, all flits of $P_{i}$ must reside in this VC in Config. . We remove $P_{i}$ from the network and label these removed packets as $P_{\text {subset }_{0}}$. We label the

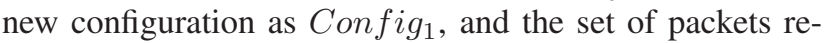
maining in this configuration as $P_{\text {subset }_{1}}$.

Step 2: We prove that when the network is routed by Alg, all packets in $P_{\text {subset }}$ can be forwarded into their current

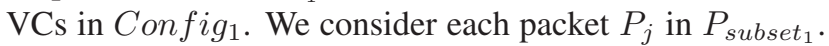
We further consider each hop hop $p_{k}$ of $P_{j}$ when the network is routed by $A l g+W P F$. Without loss of generality, we assume the head flit of $P_{j}$ is forwarded from $V C_{k}$ to $V C_{k+1}$ during $h o p_{k}$. There are two situations for $V C_{k+1}$.

2.1) $V C_{k+1}$ is empty when the head flit of $P_{j}$ is forwarded into it; therefore, $V C_{k+1}$ is allocated to $P_{j}$ using conservative $\mathrm{VC}$ re-allocation. Thus, if the network is routed by $A l g, P_{j}$ can use $V C_{k+1}$.

2.2) $V C_{k+1}$ is not empty when the head flit of $P_{j}$ is forwarded into it, thus $V C_{k+1}$ is allocated to $P_{j}$ using WPF. Since $P_{j}$ can be forwarded into $V C_{k+1}$, the routing algorithm allows $P_{j}$ to use $V C_{k+1}$. However, if the network is routed by $\mathrm{Alg}, P_{j}$ cannot be forwarded into $V C_{k+1}$ until it is empty. Since $A l g$ is deadlock-free, the packet currently residing in $V C_{k+1}$ must be sent out in a limited time. Then $V C_{k+1}$ can be re-allocated to $P_{j}$ using conservative VC reallocation. Thus, if the network is routed by $\mathrm{Alg}, P_{j}$ can use $V C_{k+1}$.

Considering 2.1) and 2.2) together, for each hop, if a VC is used by $P_{j}$ when the network is routed by $A l g+W P F$, this $\mathrm{VC}$ can be also used by $P_{j}$ when the network is routed by $A l g$. Thus, $P_{j}$ can be routed to its current $\mathrm{VC}(\mathrm{s})$ in Config 1 by Alg.

Step 3: We prove that Config $_{1}$ is a deadlock configuration for $A l g$. For each $P_{i}$ in the removed packet set $P_{\text {subset }}$, all flits of $P_{i}$ reside in one $\mathrm{VC}$ but the head flit of $P_{i}$ is not at the head of its VC. Thus, removing $P_{i}$ from the network does not create an empty VC; each VC now holds flits of only one packet. Alg utilizes conservative $\mathrm{VC}$ re-allocation which only allows empty VCs to be re-allocated. Therefore all packets in the remaining packet set $P_{\text {subset }_{1}}$ still wait for VCs held by other packets in $P_{\text {subset }}$. Thus, Config 1 is a deadlock configuration for $A l g$, but $A l g$ is deadlock-free, so there is no such deadlock configuration. Thus, $A l g+W P F$ is deadlock-free as well.

Note that our proof does not make any assumption about the routing algorithm; WPF can be utilized with any fully adaptive routing algorithm if it is deadlock-free using conservative $\mathrm{VC}$ re-allocation. WPF removes the constraints of conservative VC re-allocation. Thus, it is an important extension of these theories.

\subsection{Fully Adaptive Routing Algorithm}

As demonstrated in Section 1, fully adaptive routing algorithms can yield worse performance than deterministic and partially adaptive ones in VC-limited networks. To combat this problem, we leverage WPF to design a novel fully adaptive routing algorithm with superior performance. Our design is based on Duato's theory $[12,13]$. In a VClimited NoC, the routing algorithm should maintain maximum routing flexibility; it should allow the use of adaptive VCs after using escape VCs. Otherwise, once a packet goes into escape VCs, it loses adaptivity in subsequent routing. The design must guarantee that at any time a packet is able to request an escape $\mathrm{VC}[12,13]$.

We make a simple modification. In port-selection-first algorithms, the only time a packet cannot use an escape VC is when the selection strategy chooses an port that violates DOR. Our design allows the packet to violate the selection in this case; the packet can apply for the escape VC of the other port that was not selected in addition to adaptive VCs of the selected one. Using $P_{1}$ in Figure 6 as an example, if the selection strategy chooses the south port, our algorithm allows $P_{1}$ to request the escape $\mathrm{VC}$ of the east output port as well. If there is only one available port, this port must adhere to DOR, and the packet can request its escape VC. Our design guarantees that a packet always has an opportunity to use an escape VC. Thus, it allows a packet to move back into an adaptive $\mathrm{VC}$ after using an escape VC. It only needs $V: 1$ arbiters in the first stage of the VC allocator. Large arbiters result in more hardware overhead and introduce additional delay on the critical path.

\subsection{Router Microarchitecture}

The pipeline of a canonical NoC Router [8, 16, 36, 40] consists of four stages: routing computation (RC), VC allocation (VA), switch allocation (SA) and switch traversal (ST). Several optimizations are applied to achieve high baseline performance. The speculative switch allocation is used to parallelize VA and SA [40]. Look-ahead routing removes $\mathrm{RC}$ from the critical path; the adaptive routing al- 
gorithm calculates at most two available output ports one hop ahead and applies a selection strategy to choose an optimal one [21, 27, 31]. The delay of the baseline router is 2 cycles plus an additional cycle for link traversal.

Both WPF and our routing algorithm only require simple modifications to the baseline $\mathrm{VC}$ allocator. They can be used with any type of VC allocator; we assume a separable $\mathrm{VC}$ allocator which is widely used due to its low complexity and high frequency $[2,36,40]$. In a separable VC allocator, each input VC determines which output VC of the selected output port to bid on in the first stage. The winning requests from the first stage then arbitrate for an output VC in the second stage. We modify the first stage arbiters.

First, we need to monitor whether a downstream VC is free to be re-allocated with WPF. The criterion is that the downstream VC holds the tail flit of its most recently allocated packet and still has enough free slots to hold the entire new packet. Calculating whether there are enough free buffer slots for a new packet introduces some hardware overhead. However, considering that cache coherence packets exhibit a bimodal distribution and long packets are generally longer that the VC depth, we focus on applying WPF to single-flit packets. Thus, if a downstream VC receives the tail flit of the most recently allocated packet, and it still has free slots, it can be re-allocated to a single-flit packet.

Figure 9 depicts our proposed VC allocator. $R e g_{0}$ records if a downstream $\mathrm{VC}$ is free to be re-allocated with conservative re-allocation; the downstream VC is currently empty. An additional register $\operatorname{Reg}_{1}$ is needed to record whether a downstream VC is free to be re-allocated with WPF. Based on the incoming packet type, $R e g_{0}$ or $R e g_{1}$ is chosen as the input to MUX0. If the incoming packet is a single-flit packet, we apply WPF, choosing the contents of $R e g_{1}$ as the input for MUX1. Otherwise, the contents of $R e g_{0}$ are sent to MUX0. Updates to $R e g_{0}$ and $R e g_{1}$ are off the critical path since a router monitors the status of downstream VCs using credits [8]. The only increase in delay for WPF is an additional 2-input multiplexer: MUX0.

To support our new fully adaptive routing algorithm, we modify MUX1 and DEMUX1, as shown in Figure 9. MUX1 needs two additional input signals: DOR and the other output port. The DOR signal indicates if the chosen optimal output port obeys DOR or not. The other output port signal records the available output port that was not chosen. The routing computation logic produces these two signals. If the DOR signal is ' 0 ', then the selected output port violates DOR path. In this case, the status of the escape VC for the other output port rather than the chosen optimal one will be sent to the $V: 1$ arbiter. This is accomplished with a 2-input multiplexer whose select signal is DOR. DEMUX1 also needs these two additional signals. If the DOR signal is ' 0 ', the result of $V: 1$ arbiter is de-multiplexed to the second stage arbiter for the escape $\mathrm{VC}$ of the other

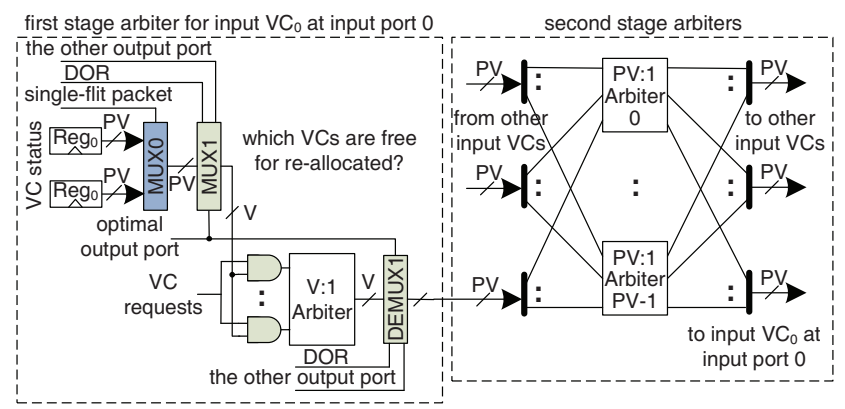

Figure 9. The proposed VC allocator for one VN.

Table 2. The critical path delay and area results.

\begin{tabular}{|l|l|l|}
\hline Design & Delay $(\mathrm{ns})$ & Area $\left(\mu \mathrm{m}^{2}\right)$ \\
\hline Port-selection-first (Figure 5(a)) & 1.78 & 49437.4 \\
\hline Naive design (Figure 5(b)) & 1.92 & 56045.4 \\
\hline Proposed design (Figure 9) & 1.79 & 49512.6 \\
\hline
\end{tabular}

output port instead of the chosen port. A 2-input demultiplexer implements this function. The increased delay for our new fully adaptive routing algorithm is an additional 2input multiplexer and demultiplexer.

To analyze the hardware overhead, we implement the three VC allocators (Figures 5 and 9) in RTL Verilog for an open-source NoC router [2] and synthesize in Synopsys Design Compiler with a TSMC 65nm standard cell library. The designs operate at $500 \mathrm{MHz}$ under normal conditions $\left(1.2 \mathrm{~V}, 25^{\circ} \mathrm{C}\right)$. We use simple round-robin arbiters [8]. This router has 5 ports $(P=5)$ and supports $4 \mathrm{VNs}$; each $\mathrm{VN}$ has 2 VCs $(V=2)$. Table 2 presents the area and critical path delay estimates. The naive design uses 4:1 arbiters in the first stage, resulting in a 7.9\% longer critical path and $13.4 \%$ more area than the port-selection-first design. Our design uses $2: 1$ arbiters in the first stage and only increases the critical path by $0.5 \%$ and area by $0.2 \%$. An allocator's power consumption is largely decided by the arbiter size $[2,50]$; given the small arbiters in our design, there should be negligible power overhead compared with the port-selection-first design. However, we omit a detailed power evaluation as it depends on the activity factor of each signal.

\section{Evaluation}

We modify the cycle-accurate Booksim simulator [8] to model the microarchitecture discussed in Section 4. We compare the performance of our proposed fully adaptive routing algorithm with conservative VC re-allocation (FULLY) and with WPF (FULLY+WPF) against several routing algorithms. We implement a port-selection-first fully adaptive routing algorithm with conservative $\mathrm{VC}$ re-allocation (PSF) and with WPF (PSF+WPF). The deterministic routing algorithm is DOR. West-first, negative-first and oddeven represent partially adaptive algorithms. Since the design of selection strategy is orthogonal to this paper, we use a local selection strategy for all adaptive algorithms; when there are two available output ports, the selection strategy 
Table 3. Baseline configuration and variations.

\begin{tabular}{|l|l|l|}
\hline Characteristic & Baseline & Variations \\
\hline Topology (mesh) & $4 \times 4$ & $8 \times 8$ \\
\hline VCs/VN & 2 & 4 \\
\hline Flit buffers/VC & 4 & 3,2 \\
\hline Packet length (flits) & long: 5, short: 1 & - \\
\hline SFP ratio & $80 \%$ & $60 \%, 40 \%$ \\
\hline Warmup cycles, Total Cycles & 10000,100000 & - \\
\hline
\end{tabular}

Table 4. Full system simulation configuration.

\begin{tabular}{|l|l|}
\hline \# of cores & 16 \\
\hline L1 cache (D \& I) & private, 4-way, 32KB each \\
\hline L2 cache & private, 8-way, 512KB each \\
\hline Cache coherence & MOESI distributed directory \\
\hline Topology & $4 \times 4$ Mesh \\
\hline
\end{tabular}

chooses the port with more free buffers.

We evaluate both synthetic traffic and real applications. For synthetic traffic patterns, we use one VN since each VN is independent. Our baseline configuration uses a $4 \times 4$ mesh with 2 VCs that are each 4 flits deep. The packet lengths exhibit a bimodal distribution; there are single-flit and five-flit packets. The baseline single-flit packet (SFP) ratio is $80 \%$. Table 3 summarizes the baseline network configuration and the variations used in the sensitivity studies.

To measure full-system performance, we leverage two simulation frameworks: FeS2 [37] for x86 simulation and BookSim for NoC simulation. FeS2 is a timing-first, multiprocessor, x86 simulator, implemented as a module for Virtutech Simics [32]. We run PARSEC benchmarks [3] with 16 threads on a 16-core CMP. We assume cores optimized for clock frequency; they are clocked at a frequency $5 \times$ higher than the network. Prior research shows the frequency of simple cores in many-core platform can be optimized to $5 \sim 10 \mathrm{GHZ}$, while the frequency of NoC router are limited by the allocator speed with a large number of VCs [11]. As we consider several VNs, more VCs are needed. Thus, it is reasonable to assume cores will be clocked faster than the network. Each core is connected to private, inclusive L1 and L2 caches. Cache lines are 64 bytes; long packets are 5 flits wide with a 16-byte flit width. We use a distributed, directory-based MOESI coherence protocol which needs 4 VNs for protocol-level deadlock freedom. Each VN has 2 VCs; each VC is 4 flits deep. All benchmarks use the simsmall input sets to reduce simulation time. The total runtime is used as the metric for full-system performance. Table 4 presents the system configuration.

\subsection{Performance of synthetic workloads}

Figure 10 illustrates the performance of several routing algorithms in our baseline configuration using four synthetic traffic patterns: bit reverse, hotspot and 2 transpose patterns. Across these four patterns, the fully adaptive routing algorithms (PSF and FULLY) show the poorest performance. Although PSF and FULLY offers adaptiveness for all traffic, conservative $\mathrm{VC}$ re-allocation significantly limits their performance. In contrast, DOR and partially adap- tive routing algorithms use aggressive $\mathrm{VC}$ re-allocation. For all four patterns, PSF's performance is worse than FULLY. PSF's performance is further limited by its poor flexibility: once a packet enters an escape VC, the packet can only be routed by DOR using escape VCs in subsequent hops.

For bit reverse traffic, a source node with bit address $\left\{s_{3}, s_{2}, s_{1}, s_{0}\right\}$ sends traffic to destination $\left\{s_{0}, s_{1}, s_{2}, s_{3}\right\}$. $62.5 \%$ of this traffic is between the north-east and southwest quadrants; negative-first offers adaptiveness for this traffic. Only $37.5 \%$ of the traffic is eastbound; west-first offers adaptiveness for this traffic, which leads to poorer performance than negative-first. The adaptiveness offered by odd-even is lower than negative-first, thus its performance is worse than negative-first. Although WPF improves the VC utilization for PSF+WPF, its saturation throughput is lower than odd-even and negative-first. PSF+WPF is still limited by its poor flexibility. FULLY+WPF provides high $\mathrm{VC}$ utilization and significant routing flexibility leading to the highest saturation throughput ${ }^{4}$.

For transpose- 1 , a node $(i, j)$ sends messages to node $(3-j, 3-i)$. Negative-first deteriorates to DOR for this pattern. West-first still offer adaptiveness for $37.5 \%$ of the traffic, thus it has better performance than negative-first. Odd-even offers greater adaptiveness than the other two partially adaptive algorithms and has higher performance. FULLY+WPF offers adaptiveness for all traffic, achieving $15.7 \%$ higher saturation throughput than odd-even.

Transpose- 2 is a favorable pattern for negative-first; a node $(i, j)$ sends messages to node $(j, i)$. Negative-first offers adaptiveness for all traffic in this pattern and has the highest performance. Although FULLY+WPF offers adaptiveness for all packets as well, its performance is limited by the restriction on usage of the escape VCs: only if the output port adheres to DOR, can the escape VC be used. The performance of FULLY+WPF and odd-even with transpose- 2 are very close to their performance with transpose- 1 since the two transpose patterns are symmetric and these two algorithms offer the same adaptiveness for them.

With hotspot traffic, four nodes are chosen as hot spots and receive an extra 20\% traffic in addition to the uniform random traffic. This pattern mimics memory controllers receiving a disproportionate amount of traffic. FULLY+WPF and odd-even algorithms show higher performance than negative-first and west-first ones, because they can offer greater adaptiveness. Due to the more limited adaptiveness offered by odd-even, its performance is worse than FULLY+WPF. DOR has better performance than negative-first and west-first, since DOR more evenly distributes uniform traffic which is used as the background in this pattern.

In summary, with conservative $\mathrm{VC}$ re-allocation, the fully adaptive algorithm has the worst performance. Negative-

\footnotetext{
${ }^{4}$ The saturation point is measured as the injection rate at which the average latency is 3 times the zero load latency.
} 


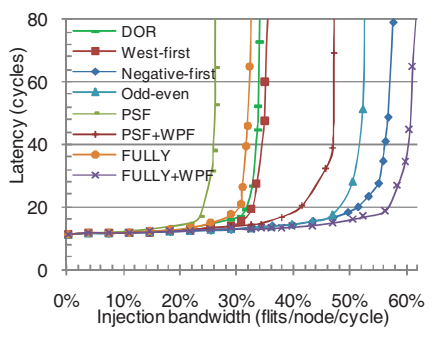

(a) Bit reverse.

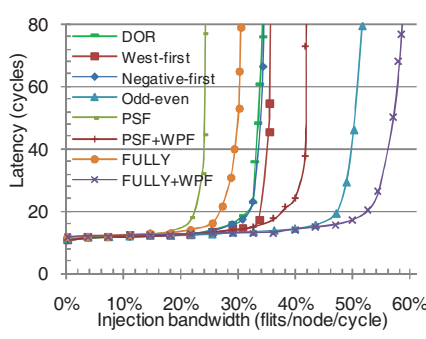

(b) Transpose-1.

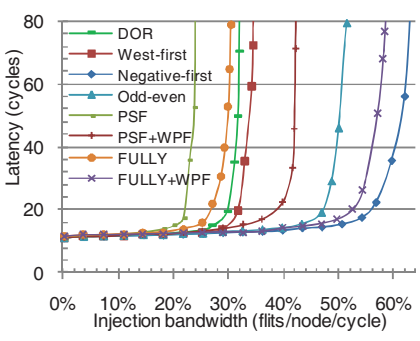

(c) Transpose-2.

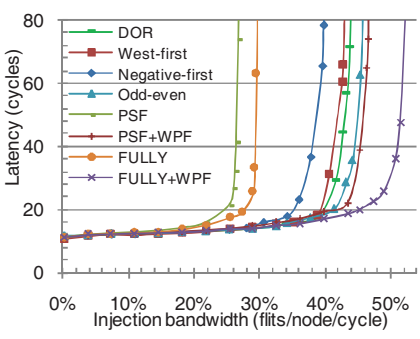

(d) Hotspot.

Figure 10. Routing algorithm performance for the baseline configuration.

Table 5. Average saturation throughput improvement.

\begin{tabular}{|l|l|l|l|}
\hline Algorithm & Improvement & Algorithm & Improvement \\
\hline FULLY & $88.9 \%$ & Odd-even & $16.3 \%$ \\
\hline DOR & $64.5 \%$ & PSF & $130.9 \%$ \\
\hline West-first & $58.6 \%$ & PSF+WPF & $31.3 \%$ \\
\hline Negative-first & $26.6 \%$ & & \\
\hline
\end{tabular}

first and west-first offer uneven adaptiveness for different patterns. For example, although negative-first achieves high performance for transpose-2, it deteriorates to DOR for transpose-1. As the traffic in NoCs changes throughout runtime and different VNs may run different traffic patterns, these partially adaptive routing algorithms are unsuitable. Odd-even offers limited adaptiveness for all traffic patterns. WPF improves VC utilization for fully adaptive routing algorithms. In a VC-limited environment, routing flexibility must also be considered to fully leverage WPF. $\mathrm{PSF}+\mathrm{WPF}$ does not provide enough routing flexibility resulting in lower performance than some partially adaptive algorithms. FULLY+WPF provides high VC utilization and routing flexibility, leading to the best performance. Table 5 gives the average saturation throughput improvement of FULLY+WPF over the other algorithms across all four patterns. The $88.9 \%$ saturation throughput gap between FULLY+WPF and FULLY reflects the effect of WPF. The gap between FULLY+WPF and PSF+WPF represents of the effect of routing flexibility; sufficient flexibility leads to an average saturation throughput improvement of $31.3 \%$.

\subsection{Performance of PARSEC benchmarks}

Figure 11 shows the speedups relative to PSF for the PARSEC workloads. We divide the 10 applications into 2 classes. For blackscholes, fluidanimate, raytrace and swaptions, different routing algorithms have similar performance. The working sets of these applications fit into the caches leading to a lightly loaded network. Their system performance is unaffected by techniques that improve throughput, such as sophisticated routing algorithms. However, the routing algorithm influences the performance of the remaining 6 applications; they exhibit bursty communication and have heavier loads. Their system performance is sensitive to network optimizations; routing algorithms with higher saturation throughputs improve performance. For example, FULLY+WPF has $48.5 \%$ and $43.0 \%$ speedup over PSF for facesim and streamcluster. West- first has the best performance for vips because most of its bursty communication is eastbound. For facesim and streamcluster, negative-first offers higher adaptiveness than odd-even for bursty communication, thus achieving higher performance. For all heavy load applications except vips, FULLY+WPF has the best performance. Across these applications, FULLY+WPF achieves an average of $21.3 \%$ and maximum $37.8 \%$ speedup over FULLY. With sufficient flexibility, FULLY+WPF has an average $12.1 \%$ speedup over PSF+WPF. The average speedups of FULLY+WPF are $29.3 \%, 15.0 \%, 10.1 \%, 9.9 \%$ and $10.4 \%$ over PSF, DOR, west-first, negative-first and odd-even, respectively. Our design supports higher saturation throughput; if higher saturation throughput is not required, WPF can benefit the NoC by reducing network resources such as reducing the buffer resources and reducing the channel width.

\section{Sensitivity to Network Design}

Individual network implementations are likely to vary from our baseline configuration, depending on the needs of the system. We explore variations for further insight. Except for the analyzed parameter, the other parameters are the same as the baseline (Table 3).

Single-flit packet ratios. Single-flit packet (SFP) ratios depend on the running application, the cache hierarchy and the coherence protocol. To test the robustness of WPF, we evaluate $60 \%$ and $40 \%$ SFP ratios for transpose- 1 traffic. As illustrated in Figure 12, DOR, west-first, negative-first and odd-even exhibit nearly identical performance for different SFP ratios. Since they apply aggressive VC re-allocation, their performance is not sensitive to packet length. However, the performance of PSF and FULLY improves as the SFP ratio shrinks. The conservative VC re-allocation used by PSF and FULLY favors long packets since they utilize buffers more efficiently than short ones. As the SFP ratio decreases so does the possibility of applying WPF. Thus, the performance gap between FULLY+WPF and FULLY (or PSF+WPF and PSF) decreases. However, even with a $40 \%$ SFP ratio, FULLY+WPF achieves a 53.1\% saturation throughput improvement over FULLY. For these different SFP ratios, FULLY+WPF has the best performance.

VC depth. Different NoCs may use different VC depths. 


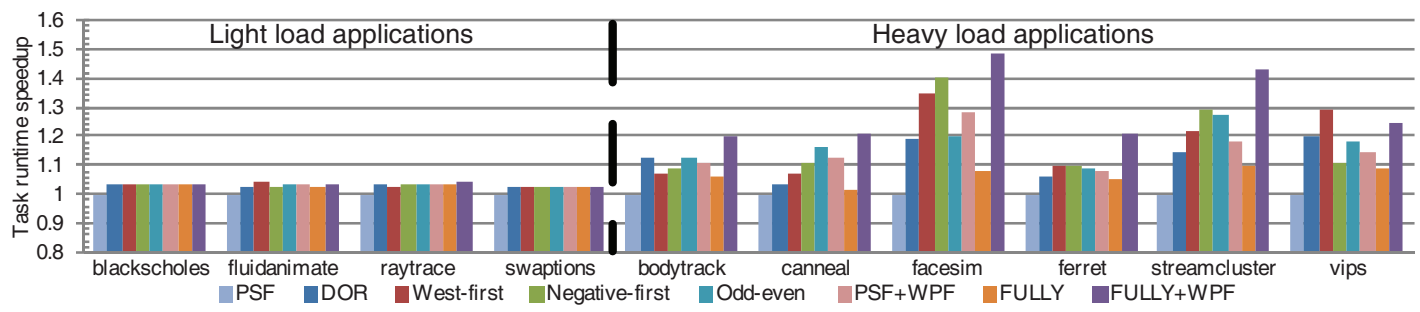

Figure 11. System speedup for PARSEC benchmarks.

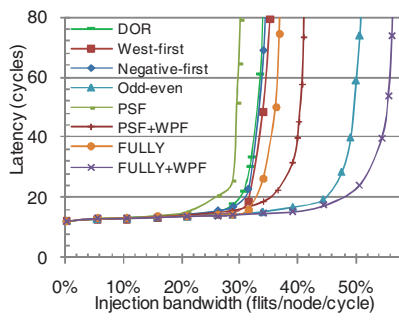

(a) $60 \%$ SFP ratio

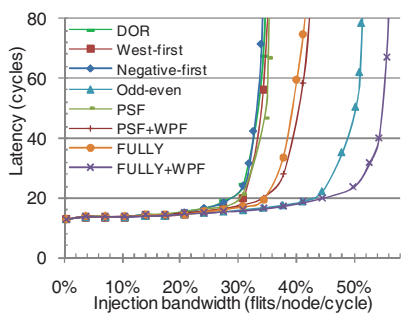

(b) $40 \%$ SFP ratio.
Figure 12. The performance with different SFP ratios.

To test the flexibility of WPF, we further evaluate 3- and 2flit deep VCs. Comparing 4 flits/VC (Figure 10(a)) and 3 flits/VC (Figure 13(a)), the performance of DOR and westfirst remain almost constant, while FULLY and PSF exhibit minor performance degradation. DOR and west-first offer no or very limited adaptiveness which is a major factor in their performance. Thus, reducing the $\mathrm{VC}$ depth from 4 to 3 has little effect. The bottleneck of FULLY and PSF is conservative $\mathrm{VC}$ re-allocation. Considering the majority of single-flit packets, reducing the $\mathrm{VC}$ depth from 4 to 3 only affects performance slightly. However, the performance of FULLY+WPF, PSF+WPF, odd-even and negative-first declines with shallower VCs since the VC depth is a bottleneck for them. Shallow VCs increase the number of hops that a blocked packet spans, which increases the effect of chained blocking [46].

Comparing 3 and 2 flits/VC, performance drops for all algorithms. FULLY has better performance than DOR and west-first with 2 flits/VC. As VC depth decreases, the difference between aggressive and conservative $\mathrm{VC}$ re-allocation declines. FULLY has a relative performance improvement. However, even with only 2 flits/VC, WPF still optimizes the performance since short packets dominate traffic. In Figure 13(b), FULLY+WPF has a $46.2 \%$ saturation throughput improvement over FULLY. Applying WPF on fully adaptive routing algorithms leads to superior performance even with half of the buffer resources; enabling the design of a very low-cost NoC. With 2 flits/VC (Figure 13(b)), FULLY+WPF achieves a saturation throughput of $40.3 \%$, while the saturation throughput of FULLY is $32.3 \%$ with 4 flit$\mathrm{s} / \mathrm{VC}$ (Figure 10(a)). The same is true for PSF+WPF with 2 flits/VC and PSF with 4 flits/VC.

VC count. As semiconductor scaling continues, a VN may be configured with more VCs. Coherence protocols

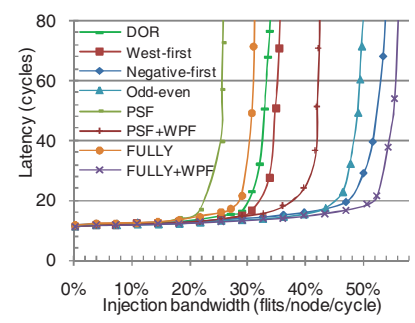

(a) 3-flit deep VCs.

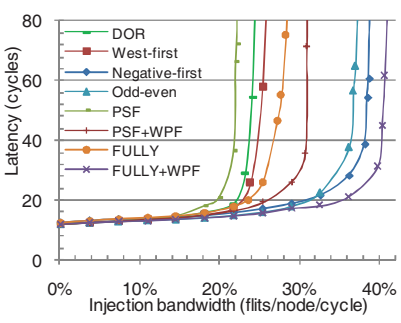

(b) 2-flit deep VCs.
Figure 13. The performance with different VC depths.

may be optimized to reduce the number of required VNs allowing more VCs per VN. Comparing Figure 14(a) (4 VC$\mathrm{s} / \mathrm{VN})$ and Figure 10(a) (2 VCs/VN), the performance of DOR, west-first and odd-even is almost the same. These algorithms offer limited adaptiveness; although additional experimental results show increasing the $\mathrm{VC}$ count from 1 to 2 improves performance, increasing the $\mathrm{VC}$ count from 2 to 4 cannot reduce the congestion for physical paths and does not further improve performance. Negative-first has a modest performance improvement. In contrast, PSF, FULLY, PSF+WPF and FULLY+WPF all have significant improvement; more VCs mitigate the negative effects of conservative $\mathrm{VC}$ re-allocation. The performance difference between PSF and FULLY (or PSF+WPF and FULLY+WPF) decreases with more VCs; more VCs reduce the possibility of using escape VCs in PSF which limits the packets that lose adaptivity.

Figure 14(b) shows the performance of transpose-2, which is a favorable pattern for negative-first. FUL$\mathrm{LY}+\mathrm{WPF}$ achieves almost the same performance as negative-first; with more VCs, the effect of restricting the use of escape VCs in FULLY+WPF declines. With more VCs, the gap between FULLY and FULLY+WPF (or PSF and PSF+WPF) diminishes. More VCs improves the possibility of a packet being forwarded into an empty VC, thus improving the performance of FULLY (or PSF). Furthermore, using WPF to forward a new packet into a non-empty VC may result in head-of-line congestion [8] and degrade the performance of FULLY+WPF (or PSF+WPF). Nevertheless, FULLY+WPF still shows an average $19.8 \%$ saturation throughput improvement over FULLY for these two patterns with $4 \mathrm{VCs}$; providing high VC utilization strongly outweighs the negative effect of HoL blocking in a VClimited environment. Yet, additional experimental results 


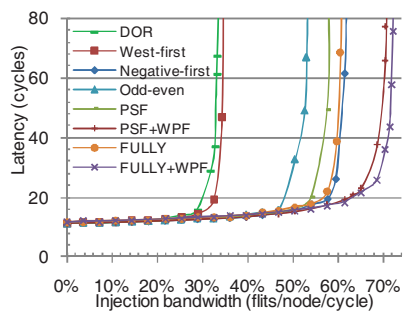

(a) Bit reverse

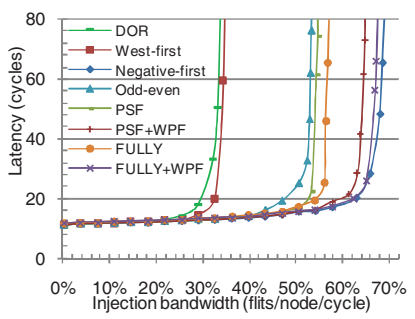

(b) Transpose-2.
Figure 14. The performance with 4 VCs/VN.

show that WPF results in minor performance degradation with $8 \mathrm{VCs} / \mathrm{VN}$ for some traffic patterns. Similarly, aggressive $\mathrm{VC}$ re-allocation slightly degrades the performance of deterministic and partially adaptive algorithms compred to conservative $\mathrm{VC}$ re-allocation with $8 \mathrm{VCs} / \mathrm{VN}$ for some patterns. With abundant VCs, the HoL blocking outweighs the positive effect of high VC utilization. Similar to VC depth, with only 2 VCs, FULLY+WPF offers near or even better performance (Figures 10(a) and 10(b)) than FULLY with 4 VCs (Figure 14) across these two patterns; WPF provides similar or higher performance with half as many VCs.

Network size. Figure 15 explores the scalability of our design for an $8 \times 8$ mesh. The trends across different algorithms are the same as with the $4 \times 4$ mesh (Figure 10). Communication is determined by the traffic pattern not the network size. Since larger networks lead to higher average hop counts [31], a larger network puts more pressure on VCs than a smaller one. WPF achieves greater performance improvement in a larger network. The average saturation throughput improvement for these two patterns of FULLY+WPF over FULLY is $108.2 \%$, while in a $4 \times 4$ mesh, it is $93.1 \%$. As packets must travel more hops in a larger network, the possibility of entering an escape VC increases. For PSF and PSF+WPF, once the packet enters an escape $\mathrm{VC}$, it loses adaptivity in subsequent hops. Therefore the performance gap between FULLY+WPF and PSF+WPF (or FULLY and PSF) increases with a larger network; providing routing flexibility becomes more important with a larger network.

In summary, although the effect of WPF decreases with smaller SFP ratios, shallower VC depths or more VCs, applying WPF with fully adaptive routing still improves performance significantly. The effect of WPF as well as routing flexibility increases with a larger network. Applying WPF to fully adaptive routing algorithms provides similar or even better performance with half of the buffer resources or VCs as a network that does not employ WPF.

\section{Further Discussion and Future Work}

Packet length and VC depth. Packet lengths for cache coherence traffic typically have a bimodal distribution. However, optimizations such as cache line compression $[11,25]$

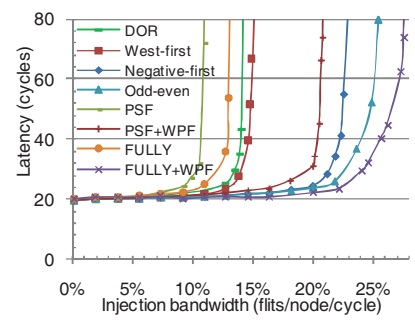

(a) Bit reverse.

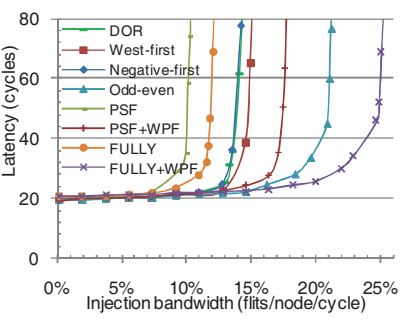

(b) Transpose- 1 .
Figure 15. The performance for an $8 \times 8$ mesh network.

create packet distributions that are not bimodal; the packet length may be distributed between a single flit and the maximum flits per packet supported by the architecture. To apply WPF on such NoCs, more downstream VC status registers are needed for the first-stage arbiters shown in Figure 9. An important consideration is how many different packet lengths to apply WPF to. The longest packet length that can use WPF is one flit shorter than the VC depth. Designers can ignore long packets, since they have few opportunities to apply WPF. This tradeoff depends on the packet length distribution, VC depth, hardware overhead and the expected performance gain. Delving into this tradeoff is left for future work. In this paper, we assume the VC depth is shorter than the maximum packet length. If the $\mathrm{VC}$ depth exceeds the maximum packet length, conservative $\mathrm{VC}$ re-allocation results in poorer VC utilization for wormhole than VCT, and applying WPF in this case behaves the same as VCT. The contribution of WPF is that it allows multiple packets to reside in one $\mathrm{VC}$ while allowing the $\mathrm{VC}$ depth to be shorter than the maximum packet length, thus giving designers more flexibility.

DAMQ and hybrid flow controls. Previous research proposed the dynamically allocated multi-queue (DAMQ) designs for both off-chip [45] and on-chip networks [38, 50] to improve the $\mathrm{VC}$ utilization. Even with DAMQ, allowing multiple packets to reside in one VC may lead to a deadlock configuration similar to Figure 3 for fully adaptive routing algorithms in a wormhole network. WPF is complimentary to DAMQ as it ensures deadlock-freedom and improves the design flexibility. WPF can viewed as a hybrid mechanism combining wormhole and VCT. There are some previous hybrid flow controls [42, 44, 30]. Hybrid switching [42] and buffered wormhole [44] remove a blocked wormhole packet to release held physical channels by utilizing either the processing node memory [42] or a central buffer [44]. Layered switching divides the long wormhole packets into several groups and tries to keep the switch allocation grants for a whole group [30]. The purpose of WPF is quite $d$ ifferent; we focus on improving the performance for fully adaptive routing algorithms in wormhole networks. 


\section{Conclusion}

Whole packet forwarding is a novel VC re-allocation scheme for fully adaptive routing algorithms in wormhole networks. This scheme allows multiple packets to reside in one VC concurrently; it greatly improves VC utilization in VC-limited networks where short packets dominate traffic. We prove that WPF does not lead to deadlock if the algorithm is deadlock-free with conservative VC reallocation. Thus, WPF is an important extension to existing deadlock-avoidance theories. We further propose a novel fully adaptive routing algorithm that exploits WPF and provides routing flexibility with modest hardware overhead. Compared with conservative VC re-allocation, WPF improves the saturation throughput by $88.9 \%$ on average in synthetic traffic patterns and achieves up to $37.8 \%(21.3 \%$ average) full-system speedup for network-intensive PARSEC benchmarks, and offers similar or even better performance with half of the buffer resources or VCs.

\section{Acknowledgments}

We thank the anonymous reviewers for their helpful suggestions and members of Prof. Enright Jerger's group for feedback. We also thank Daniel Becker of Stanford for his explanation on the open-source router implementation. This work is supported by the University of Toronto, NSERC of Canada, the Connaught Fund, 863 Program of China (2012AA010302), NSFC (61070037, 61025009, 60903039, 61103016), China Edu. Fund. (20094307120012), Hunan Prov. Innov. Fund. For PostGrad. (CX2010B032).

\section{References}

[1] K. Anjan and T. Pinkston. An efficient, fully adaptive deadlock recovery scheme: Disha. In ISCA 1995.

[2] D. Becker and W. Dally. Allocator implementations for network-onchip routers. In SC 2009.

[3] C. Bienia et al. The parsec benchmark suite: characterization and architectural implications. In PACT 2008

[4] G.-M. Chiu. The odd-even turn model for adaptive routing. IEEE Trans. Parallel Distrib. Syst., 11(7):729 -738, July 2000.

[5] W. Dally. Virtual-channel flow control. IEEE Trans. Parallel Distrib. Syst., 3(2):194 -205, Mar 1992.

[6] W. Dally and C. Seitz. Deadlock-free message routing in multiprocessor interconnection networks. IEEE Trans. Comp., C-36(5):547 -553 , May 1987.

[7] W. Dally and B. Towles. Route packets, not wires: on-chip interconnection networks. In DAC 2001.

[8] W. Dally and B. Towles. Principles and Practices of Interconnection Networks. Morgan Kaufmann, San Francisco, CA, USA, 2003.

[9] W. Dally and C. Seitz. The Torus routing chip. Distributed Computing, 1:187-196, 1986

[10] R. Das et al. Design and evaluation of a hierarchical on-chip interconnect for next-generation CMPs. In HPCA 2009.

[11] R. Das et al. Performance and power optimization through data compression in network-on-chip architectures. In HPCA 2008.

[12] J. Duato. A new theory of deadlock-free adaptive routing in wormhole networks. IEEE Trans. Parallel Distrib. Syst., 4(12):1320 1331, December 1993.

[13] J. Duato. A necessary and sufficient condition for deadlock-free adaptive routing in wormhole networks. IEEE Trans. Parallel Distrib. Syst., 6(10):1055 -1067, 1995.

[14] J. Duato. A necessary and sufficient condition for deadlock-free routing in cut-through and store-and-forward networks. IEEE Trans. Parallel Distrib. Syst., 7(8):841 -854, August 1996.

[15] J. Duato and T. Pinkston. A general theory for deadlock-free adaptive routing using a mixed set of resources. IEEE Trans. Parallel Distrib. Syst., 12(12):1219-1235, dec 2001.
[16] N. Enright Jerger and L. Peh. On-Chip Networks. Morgan and Claypool Publishers, 2009.

[17] C. Fallin et al. CHIPPER: A low-complexity bufferless deflection router. In HPCA 2011.

[18] E. Fleury and P. Fraigniaud. A general theory for deadlock avoidance in wormhole-routed networks. IEEE Trans. Parallel Distrib. Syst., 9:626-638, July 1998.

[19] B. Fu et al. An abacus turn model for time/space-efficient reconfigurable routing. In ISCA 2011.

[20] C. Glass and L. Ni. The turn model for adaptive routing. In ISCA 1992.

[21] P. Gratz, B. Grot, and S. Keckler. Regional congestion awareness for load balance in networks-on-chip. In HPCA 2008.

[22] P. Gratz et al. On-chip interconnection networks of the TRIPS chip. Micro, IEEE, 27(5):41 -50, Sept.-Oct. 2007.

[23] M. Hayenga et al. SCARAB: A single cycle adaptive routing and bufferless network. In MICRO 2009.

[24] J. Hu and R. Marculescu. DyAD - smart routing for networks-onchip. In DAC 2004.

[25] Y. Jin et al. Adaptive data compression for high-performance lowpower on-chip networks. In MICRO 2008.

[26] P. Kermani and L. Kleinrock. Virtual cut-through: a new computer communication switching technique. Computer Networks, 1979

[27] J. Kim et al. A low latency router supporting adaptivity for on-chip interconnects. In DAC 2005.

[28] M. Li et al. DyXY - a proximity congestion-aware deadlock-free dynamic routing method for network on chip. In DAC 2006.

[29] X. Lin et al. The message flow model for routing in wormholerouted networks. IEEE Trans. Parallel Distrib. Syst., 6(7):755 -760, Jul 1995.

[30] Z. Lu et al. Layered switching for networks on chip. In DAC 2007.

[31] S. Ma et al. DBAR: an efficient routing algorithm to support multiple concurrent applications in networks-on-chip. In ISCA 2011.

[32] P. S. Magnusson et al. Simics: A full system simulation platform. Computer, 35:50-58, February 2002.

[33] R. Marculescu et al. Outstanding research problems in NoC design: system, microarchitecture, and circuit perspectives. IEEE Trans. Comp.-Aided Des. Integ. Cir. Sys., 28:3-21, January 2009.

[34] M. M. K. Martin et al. Multifacet's general execution-driven multiprocessor simulator (gems) toolset. SIGARCH Comput. Archit. News, 33:92-99, November 2005.

[35] S. Mukherjee et al. The Alpha 21364 network architecture. In Hot Interconnects 2001.

[36] R. Mullins et al. Low-latency virtual-channel routers for on-chip networks. In ISCA 2004.

[37] N. Neelakantam et al. FeS2: A full-system execution-driven simulator for x86. In Poster presented at ASPLOS 2008.

[38] C. Nicopoulos et al. ViChar: A dynamic virtual channel regulator for network-on-chip routers. In MICRO 2006.

[39] U. Y. Ogras et al. Key research problems in noc design: a holistic perspective. In CODES+ISSS 2005

[40] L.-S. Peh and W. Dally. A delay model and speculative architecture for pipelined routers. In HPCA 2001.

[41] L. Schwiebert and D. N. Jayasimha. A necessary and sufficient condition for deadlock-free wormhole routing. J. Parallel Distrib. Comput., 32:103-117, January 1996.

[42] K. Shin and S. Daniel. Analysis and implementation of hybrid switching. In ISCA 1995

[43] A. Singh et al. GOAL: a load-balanced adaptive routing algorithm for torus networks. In ISCA 2003.

[44] C. B. Stunkel et al. The SP2 high-performance switch. IBM Syst. J. 34:185-204, April 1995.

[45] Y. Tamir and G. Frazier. High-performance multiqueue buffers for VLSI communication switches. In ISCA 1988.

[46] A. Vaidya et al. Impact of virtual channels and adaptive routing on application performance. IEEE Trans. Parallel Distrib. Syst. 12(2):223 -237, Feb 2001.

[47] F. Verbeek and J. Schmaltz. A comment on "a necessary and sufficient condition for deadlock-free adaptive routing in wormhole networks". IEEE Trans. Parallel Distrib. Syst., 22(10):1775 -1776, oct. 2011

[48] F. Verbeek and J. Schmaltz. On necessary and sufficient conditions for deadlock-free routing in wormhole networks. IEEE Trans. Parallel Distrib. Syst., 22(12):2022 -2032, dec. 2011.

[49] D. Wentzlaff et al. On-chip interconnection architecture of the TILE processor. Micro, IEEE, 27(5):15 -31, Sept.-Oct. 2007.

[50] Y. Xu et al. Simple virtual channel allocation for high throughput and high frequency on-chip routers. In HPCA 2010. 